\title{
SOLVENT-FREE ACETYLATION OF CNFS AND CMFS USING DIFFERENT
}

\section{CATALYSTS}

\author{
SEONGSU PARK, ${ }^{*}$ BYUNG-DAE PARK ${ }^{*}$ and YOUNG HOON JUNG ${ }^{* *}$ \\ "Department of Wood Science and Technology, Kyungpook National University, \\ Daegu, 41566, Republic of Korea \\ ** School of Food Science and Biotechnology, Kyungpook National University, \\ Daegu, 41566, Republic of Korea \\ $₫$ Corresponding author: Byung-Dae Park, byungdae@knu.ac.kr
}

Dedicated to Professor Cristofor I. Simionescu, on his $100^{\text {th }}$ birth anniversary

\begin{abstract}
Nanocellulose has several advantages, including hydrophilicity, low density, high strength property, and large specificsurface area. However, its hydrophilicity becomes a problem when it comes in contact with a polymer matrix in nanocomposites. Therefore, the transformation of hydrophilicity into hydrophobicity through chemical modifications is important. This study focused on the impacts of two solvent-free acetylation methods using iodine (Method 1) and sulfuric acid (Method 2) as catalysts. Moreover, herein, the characteristics of acetylated cellulose nanofibrils (A-CNFs) and cellulose microfibrils (A-CMFs) produced via an aqueous counter collision (ACC) are compared. The degree of substitution (DS) of acetylated samples increased with the reaction time. Furthermore, the DS of Method 2 was higher than that of Method 1. After acetylation, the X-ray diffraction patterns of the samples were semicrystalline and amorphous owing to the destruction of crystalline cellulose. Moreover, the crystallinity of all acetylated samples decreased. Excluding a few cases of Method 2, the thermal stability increased after acetylation. Excluding the CMFs treated with Method 2, the contact angle increased with the reaction time. This indicates that the samples were transformed to exhibit hydrophobic surfaces. In addition, the cases of Method 1 exhibited higher crystallinity, thermal stability, and wettability than those of Method 2.
\end{abstract}

Keywords: solvent-free acetylation, $\mathrm{CNF}, \mathrm{CMFs}$

\section{INTRODUCTION}

Nanocellulose is a cellulose particle with at least one nanoscale dimension (1-100 nm), which includes cellulose whiskers, cellulose nanocrystals (CNC), nanofibrillated cellulose (NFC), also known as cellulose nanofibrils (CNFs), cellulose microfibrils (CMFs) or cellulose nanofibers. Regardless of its types, nanocellulose exhibits several advantages, such as low density (around 1.6 $\mathrm{g} / \mathrm{cm}^{3}$ ), high strength, large specific-surface area, and hydrophilicity. Particularly, cellulose nanofibrils exhibit long, flexible, entangled networks, as well as crystalline and amorphous domains. ${ }^{1,2}$ However, the hydrophilicity of nanocellulose causes incompatibility of CNFs and CMFs with hydrophobic polymer matrices. Therefore, the transformation of hydrophilicity into hydrophobicity via surface modification is important. Commonly, nanocellulose exhibits a large surface area with abundant hydroxyl groups. Moreover, it can be utilized for surface modifications, such as acetylation, silylation, and carbonylation. ${ }^{3}$

Acetylation, which is a common chemical modification, has the ability to transform cellulose hydrophilicity into hydrophobicity. In general, acetylation can be performed using two methods. ${ }^{4,5}$ The first one is heterogeneous acetylation, in which the cellulose is acetylated by non-solvents, such as toluene and benzene. This process has some advantages, such as insolubility and conservation of the morphological structure of the cellulose fiber. The second method is homogeneous acetylation, in which the cellulose is acetylated by a solvent and catalyst. The product of this process is soluble and 
deconstructs the crystalline network. However, some problems can be encountered when using solvents, such as the dilution of reagents and reduced reaction rate. Moreover, the use of solvents can increase the production cost, owing to additional procedures for separation and recovery. Organic solvents are also considered as hazardous compounds, particularly for humans and the environment.

In solvent-free acetylation, acetic anhydride is generally used as reagent with a catalyst. There are also various catalysts that are utilized for solventfree acetylation, including iodine, sulfuric acid, and pyridine. Lepetit et al. ${ }^{6}$ used the iodine catalytic system for microfibrillated cellulose. Eranna and Pandey ${ }^{7}$ reported about the solvent-free acetylation of rubberwood with acetic and butyric anhydrides using the iodine catalytic system. Boustani et al. ${ }^{8}$ utilized sulfuric acid as a catalyst for solvent-free acetylation with different lignocellulosic fibers. In addition, Sun et al. ${ }^{9}$ compared pyridine and its derivatives catalytic system with wheat straw. There have been various reports on neat acetylation and solvent-free acetylation with nanocellulose. ${ }^{6,10-}$ ${ }^{16}$ However, to the best of the authors' knowledge, studies that compare the solvent-free acetylation of CNFs (A-CNFs) and CMFs (A-CMFs) with different catalysts are scarce. Thus, in this work, we acetylated CNFs and CMFs produced via aqueous counter collision (ACC) ${ }^{2}$ using two solvent-free acetylation methods. In all the solvent-free acetylation methods, acetic anhydride was used as a reagent. In Method 1, iodine was used as a catalyst, whereas in Method 2, sulfuric acid was utilized. Various techniques, such as attenuated total reflection (ATR-IR) spectroscopy, X-ray diffraction (XRD), thermogravimetric analysis (TGA), and contact angle (CA) measurement, were conducted to obtain the degree of substitution (DS), crystallinity, thermal stability, and wettability.

\section{EXPERIMENTAL Materials}

Both CNFs (1 wt\%) and CMFs (1 wt \%) were purchased from CNNT, Korea, and used for solvent-free acetylation. A Whatman filter paper (Grade 1, GE Healthcare Life Sciences, UK), with a diameter of 110 $\mathrm{mm}$, was utilized for the filtration of CNFs and CMFs. Acetic anhydride (93\%) was purchased from Duksan, Korea, and utilized as a reagent for solvent-free acetylation. Iodine (99\%) and sulfuric acid (98\%) were purchased from Daejung Chemical, Korea, and used as catalysts for Method 1 and Method 2, respectively. Sodium thiosulfate anhydrous $(95 \%)$ was also purchased from Daejung Chemical, Korea. Sodium hydrogen carbonate (99\%, Daejung Chemical, Korea) and ethanol (94\%, Duksan, Korea) were utilized for washing the ACNFs and A-CMFs in Method 1 and Method 2.

\section{Solvent-free acetylation of CNFs and CMFs}

Before performing solvent-free acetylation, all CNFs and CMFs were filtered using a filter paper. Acetylation of CNFs and CMFs was performed using two different methods. Moreover, the reaction times were fixed at 0.5 , 1 , and $2 \mathrm{~h}$. All acetylations were conducted at room temperature. In Method 1, iodine was used as a catalyst according to the method reported by Lepetit et al., ${ }^{6}$ with some modification. Briefly, we used twice the amount of acetic anhydride (11.6 mL/g of CNFs and CMFs) of the original method and then stirred the reagent and the catalyst. In this catalytic system, iodine $(0.1 \mathrm{eq})$ was dissolved in acetic anhydride (10 eq). CNFs and CMFs ( $1 \mathrm{~g}$ each) were added to the mixture, and the mixture was then sealed with an aluminum foil, so as to avoid any effect of anhydride hydrolysis. After the reaction, the saturated sodium thiosulfate solution was utilized to change the color of the mixture from dark brown to white, indicating the transformation of iodine into iodide. Subsequently, the mixture was mixed and stirred with ethanol before filtration. Then, the filtered mixture was washed with three solvents: saturated sodium bicarbonate solution, distilled water, and ethanol. Lastly, the A-CNFs and A-CMFs were oven-dried at $60{ }^{\circ} \mathrm{C}$ overnight. In Method 2, sulfuric acid was utilized as a catalyst according to the method reported by Boustani et al., ${ }^{8}$ with some modification. Briefly, sulfuric acid (10 $\mu \mathrm{L})$ was blended with acetic anhydride $(10 \mathrm{~mL} / \mathrm{g}$ of CNFs and CMFs) for a few seconds. Subsequently, CNFs and CMFs (1 g each) were added to the mixture without stirring, and the mixture was then sealed with aluminum foil, similarly to Method 1 . After the reaction, the mixture was filtered and washed with ethanol and then centrifuged twice at $10000 \mathrm{rpm}$ for $10 \mathrm{~min}$. Next, the mixture was washed with distilled water and neutralized with saturated sodium bicarbonate solution. Finally, the A-CNFs and A-CMFs were freeze-dried (FDA8508, ilShin Bio Base Co., Ltd, Gyeonggido, Korea).

\section{Infrared spectroscopy}

Attenuated total reflection (ATR)-infrared (IR) measurements were obtained via IR spectroscopy (ALPHA-P model, Bruker Optics GmbH Ettlingen, Germany, equipped with an ATR universal accessory to check the functional groups of neat and acetylated CNFs and CMFs). For neat CNFs and CMFs, all the samples were oven-dried at $105{ }^{\circ} \mathrm{C}$ for $24 \mathrm{~h}$ and then scanned for 32 times in the range of $400-4000 \mathrm{~cm}^{-1}$ at a $4 \mathrm{~cm}^{-1}$ resolution. Using min-max normalization, the spectrum of each sample was then normalized.

\section{Degree of substitution}

The DS of A-CNFs and A-CMFs was calculated using the ATR-IR spectra according to the method 
reported by $\mathrm{Li}$ et $a l .{ }^{10}$ As reported by $\mathrm{Li}$ et al., a proportional relationship exists between the absorbance ratio and DS, expressed as follows:

$A_{R}=\varepsilon \times D S$

where $A_{R}$ and $\varepsilon$ denote the absorbance and absorptivity ratios of the bands at $\sim 1740 \mathrm{~cm}^{-1}$ and $1164^{-1}$, respectively, and $\varepsilon$ denotes a constant. The $D S$ denotes the average number of the acetyl group per anhydroglucose unit. In this paper, the band area was utilized for the calculation of $A_{R}$, which had a band region from 2000 to $750 \mathrm{~cm}^{-1}$. Moreover, the region from 2000 to $750 \mathrm{~cm}^{-1}$ was used for baseline correction. In calculating $A_{R}$ with the band area, the regions from 1759 to $1714 \mathrm{~cm}^{-1}$ and from 1181 to $1146 \mathrm{~cm}^{-1}$ were determined for the bands at $\sim 1740$ and $1164 \mathrm{~cm}^{-1}$, respectively. The band range and baseline are presented in Figure 1. To calculate the DS with the use of the band area, the below equation was employed:

$A_{R}=1.24 \times D S-0.40$

\section{XRD analysis}

The crystallinity of the neat CNFs and CMFs, A$\mathrm{CNF}$ and A-CMFs was calculated via XRD (D/Max2500, Rigaku MiniFlex II, Japan), with a $\mathrm{Cu} \mathrm{K} \alpha$ radiation source $(\lambda=0.15406 \mathrm{~nm})$. In addition, $\mathrm{Cu} \mathrm{K \alpha}$ radiation was generated at $40 \mathrm{kV}$ and $200 \mathrm{~mA}$. Moreover, the scan range was from $2^{\circ}$ to $50^{\circ}$, with a step of
$0.02 \% \mathrm{~min}$, and the scan speed was $2 \% \mathrm{~min}$. The neat $\mathrm{CNFs}$ and CMFs were oven-dried at $105^{\circ} \mathrm{C}$ for $24 \mathrm{~h}$ and then grounded.

The XRD patterns were used to calculate the crystallinity of the samples according to the peak deconvolution method with a Gaussian function. ${ }^{17}$ Using the OriginPro 9.0 software (OriginLab Corporation, Northampton, MA, USA), deconvolution was performed. Curve fitting was employed to extract individual crystalline and amorphous peaks. In the acetylated samples, we assumed that $2 \theta=8^{\circ}, 10^{\circ}, 13^{\circ}, 17^{\circ}$, and $22^{\circ}$ are associated with crystalline domains and that $2 \theta=$ $17^{\circ}-18^{\circ}$ corresponds to the amorphous contribution. ${ }^{11}$ In the case of the neat $\mathrm{CNFs}$ and $\mathrm{CMFs}$, the amorphous contribution was assumed to be $2 \theta=21.5^{\circ} .{ }^{18}$ Examples of calculation with a Gaussian function are presented in Figure 2. In addition, iterations were repeated until the maximum $F$ number was obtained.

The equation for calculating the crystallinity of all the samples is as follows:

Crystallinity $(\%)=\frac{S_{c}}{S_{\varepsilon}} \times 100$

where $S_{c}$ and $S_{t}$ denote the crystalline domain area and the total area of the complete domain (crystalline and amorphous), respectively.

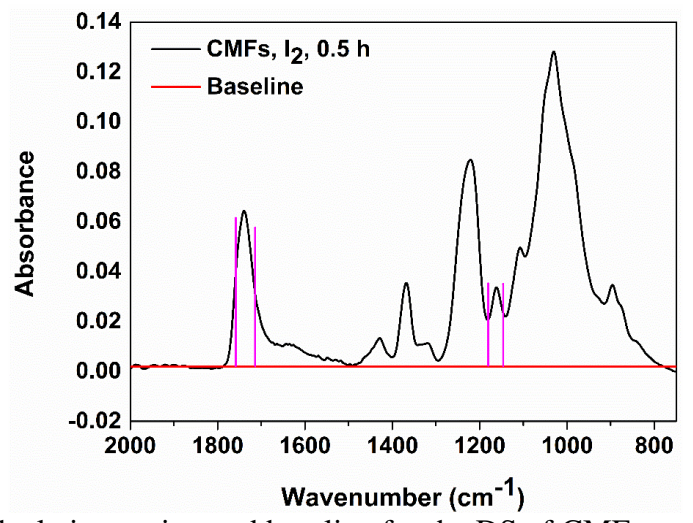

Figure 1: Typical calculating region and baseline for the DS of CMFs treated with $\mathrm{I}_{2}$ for $0.5 \mathrm{~h}$
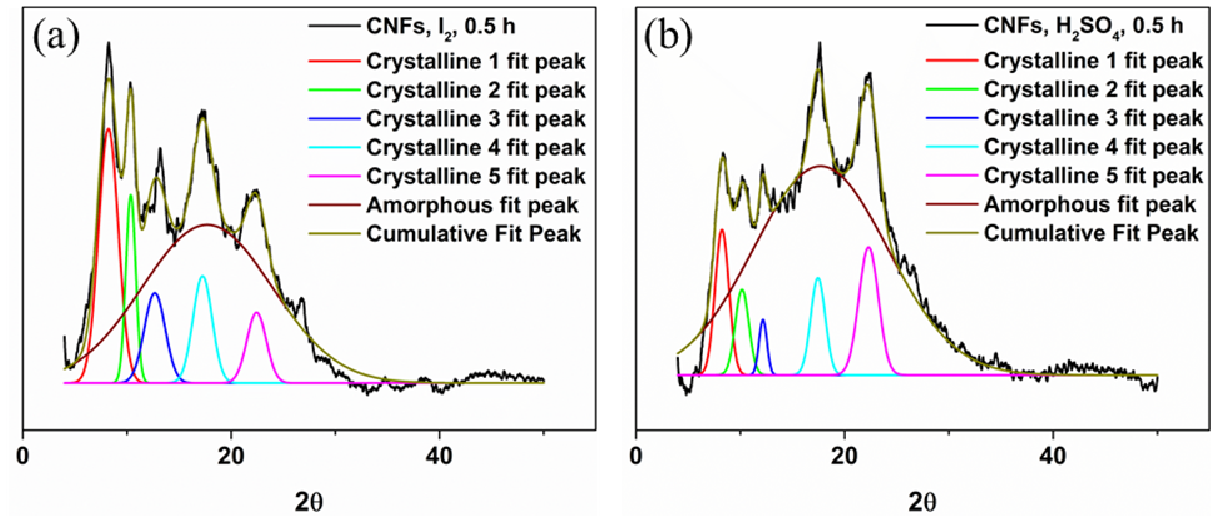

Figure 2: Examples of calculation with a Gaussian function of CNFs treated by (a) Method 1 and (b) Method 2 
Thermogravimetric analysis (TGA)

The thermal stability of the neat CNFs and CMFs, ACNFs and A-CMFs was measured via TGA (Discovery SDT 650, TA Instruments, USA). The samples (4 mg) were heated from $30{ }^{\circ} \mathrm{C}$ to $600{ }^{\circ} \mathrm{C}$ at $10{ }^{\circ} \mathrm{C} / \mathrm{min}$ under a nitrogen flow rate of $40 \mathrm{~mL} / \mathrm{min}$.

\section{Contact angle}

The static CA of the A-CNFs and A-CMFs was measured using DCAT 25 (DataPhysics Instruments, Germany) at room temperature and $65 \% \mathrm{RH}$. To prepare samples for CA, A-CNFs and A-CMFs ( $0.1 \mathrm{~g}$ each) were put in a $1 \times 1 \mathrm{~cm}$ frame and pressed at $25 \mathrm{kgf}$ for $1 \mathrm{~min}$ using hot pressure (DYMSP-100, Daeyang H.T.M Co., LTD, Korea). The CA was measured $20 \mathrm{~s}$ after dropping $10 \mu \mathrm{L}$ of distilled water on the sample surface; the measurement was repeated three times in different locations on the surface of the sample.

\section{RESULTS AND DISCUSSION ATR-IR analysis}

ATR-IR spectroscopy was employed to characterize the chemical structure of the neat $\mathrm{CNFs}$ and CMFs, A-CNFs and A-CMFs. Figure 3 presents the spectra of the neat A-CNFS and A$\mathrm{CMFs}$, and detailed information on each band are given in Table 1. A broad band around 3200-3300 $\mathrm{cm}^{-1}$ was assigned to $\mathrm{OH}$ stretching and the lowering of this band means that hydroxyl groups are substituted with acetyl groups. ${ }^{5,19}$ Popescu et $a l .{ }^{5}$ reported that the region between 1900 and 850 $\mathrm{cm}^{-1}$ was considered as a "finger print" region. Moreover, numerous well-defined peaks have been observed. Especially, after acetylation, absorption bands at 1730-1740, 1360-1370, and 1210-1220 $\mathrm{cm}^{-1}$ appeared and were assigned to $\mathrm{C}=\mathrm{O}$ stretching of ester, bending of $-\mathrm{CH}$ and $-\mathrm{CH}_{3}$, and $\mathrm{C}-\mathrm{O}$ stretching of the acetyl group, respectively. These three peaks were considered as the characteristic peaks of the acetyl group when compared with the neat sample groups. ${ }^{5,10,12,13}$

Moreover, a new peak located at 1570-1580 $\mathrm{cm}^{-1}$ has been observed for the acetylated CNFs with sulfuric acid,. This peak was assigned to the $\mathrm{COO}^{-}$groups. ${ }^{20}$ The appearance of this peak is associated with the sulfate group. In Method 2, sulfuric acid was utilized as a catalyst. Although the amount of sulfuric acid was very low, it can cause acid hydrolysis.
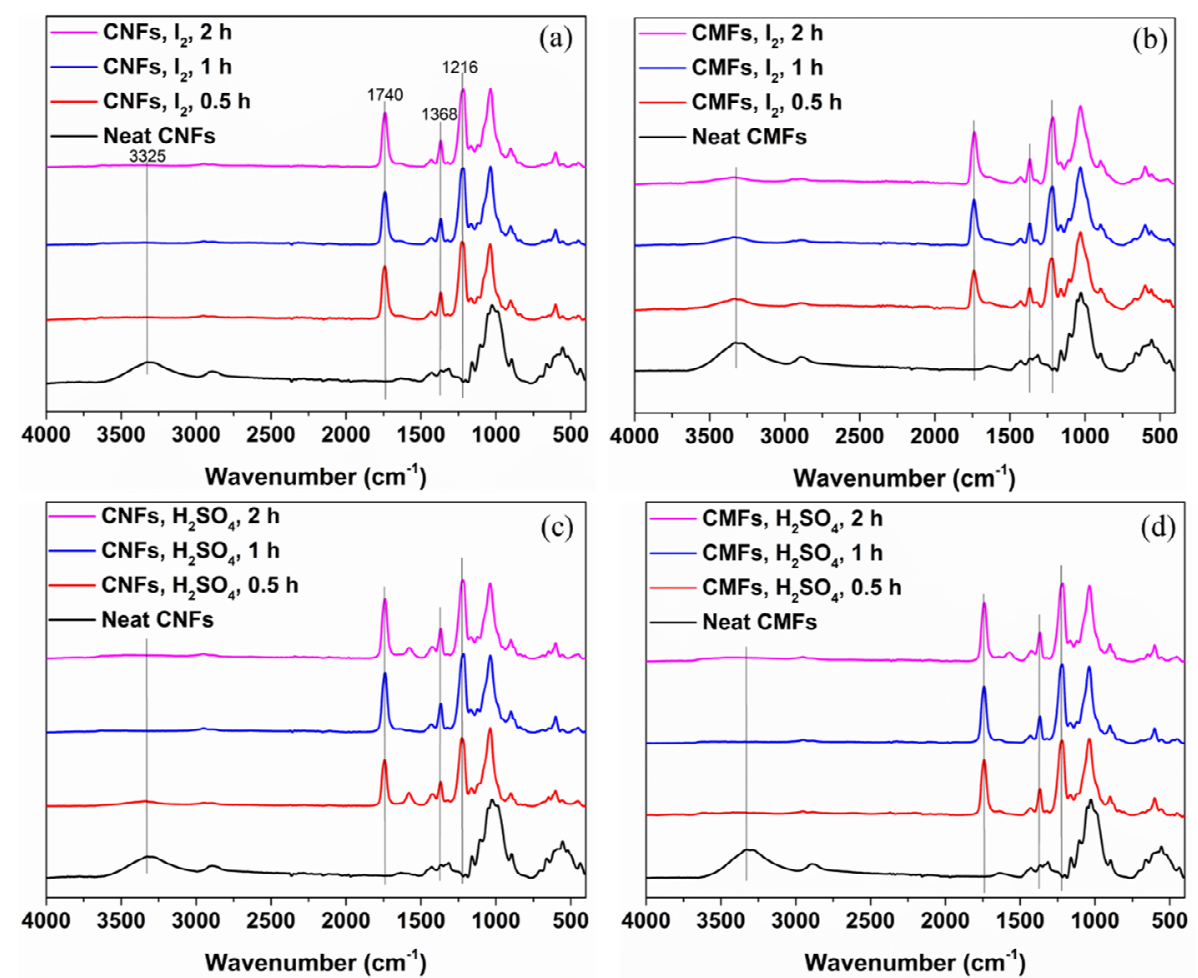

Figure 3: ATR-IR spectra of (a) CNFs treated with Method 1, (b) CMFs treated with Method 1, (c) CNFs treated with Method 2, (d) CMFs treated with Method 2

Even if washing and centrifugation were conducted

in Method 2, some sulfate groups remained due to 
the formation of ester groups. ${ }^{21}$ Furthermore, since the formation of the $\mathrm{COO}^{-}$group is a reversible reaction, it may not occur in some spectra, even if the same catalyst is used.

\section{Degree of substitution}

The DS was roughly estimated via ATR-IR analysis. ${ }^{10}$ All DS are presented in Figure 4 . As the reaction time increased, the overall value of DS also increased. Method 2 exhibited higher DS values than Method 1, a finding that may be associated with acid hydrolysis. ${ }^{21}$ As Method 2 used sulfuric acid as a catalyst, acid hydrolysis could remove the amorphous parts of CNFs and CMFs, which had larger surface area than those of Method 1. In Method 1, the DS value of CNFs was higher than that of CMFs. Kose et al. reported that as the number of the treatments was increased, the surface area of cellulose treated with ACC also increased. ${ }^{23}$ Therefore, CNFs have larger surface area than CMFs, indicating that their hydroxyl groups are more accessible than in CMFs. In other words, the greater the surface area, the easier the hydroxyl group is substituted with acetyl groups. In Method 2, CMFs exhibited a higher DS value than CNFs.

Table 1

Band assignments of ATR-IR spectra of the neat A-CNFs and A-CMFs

\begin{tabular}{|c|c|c|}
\hline Wavenumber $\left(\mathrm{cm}^{-1}\right)$ & Group & Ref. \\
\hline 896 & $\begin{array}{l}\beta \text {-glycosidic linkage between the sugar units in } \\
\text { celluloses }\end{array}$ & {$[5]$} \\
\hline 985 & $\mathrm{C}-\mathrm{O}$ stretching vibration & [5] \\
\hline $1028-1038$ & $\mathrm{C}-\mathrm{O}$ stretching ring in cellulose & [5] \\
\hline 1050 & $\mathrm{C}-\mathrm{O}$ stretching in $\mathrm{C}-\mathrm{O}-\mathrm{C}$ glycosidic bond $\mathrm{d}^{22}$ & [5], [22] \\
\hline $1100-1120$ & $\mathrm{C}-\mathrm{OH}$ stretching vibration & [5] \\
\hline $1160-1165$ & $\mathrm{C}-\mathrm{O}-\mathrm{C}$ antisymmetric stretching & {$[5],[22]$} \\
\hline $1210-1220 *$ & $\mathrm{C}-\mathrm{O}$ stretching of the acetyl group & {$[10],[22]$} \\
\hline $1360-1370 *$ & $-\mathrm{CH}$ and $-\mathrm{CH}_{3}$ bending & [10] \\
\hline $1420-1430$ & $-\mathrm{CH}_{2}$ scissors vibration & [19] \\
\hline $1570-1580$ & $\mathrm{COO}^{-}$groups & [20] \\
\hline $1630-1640$ & $\mathrm{H}-\mathrm{O}-\mathrm{H}$ bending of absorbed water & {$[5],[10],[22]$} \\
\hline $1730-1740^{*}$ & $\begin{array}{c}\mathrm{C}=\mathrm{O} \text { stretching of ester, unconjugated ketones, and } \\
\text { carbonyl groups }\end{array}$ & {$[5]$} \\
\hline $2800-2900$ & $\begin{array}{c}-\mathrm{CH}_{2} \text { asymmetric stretching and }-\mathrm{CH}_{3} \text { symmetric } \\
\text { stretching }\end{array}$ & [5], [22] \\
\hline $3200-3300$ & $\begin{array}{l}\text {-OH stretching of intramolecular and } \\
\text { intermolecular hydrogen bonds }\end{array}$ & [5], [22] \\
\hline
\end{tabular}

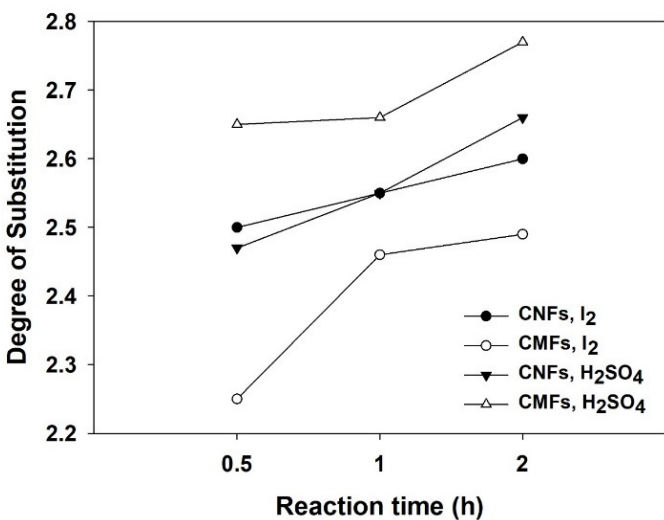

Figure 4: DS of CNFs and CMFs treated with different solvent-free acetylation methods

This finding is associated with the surface area and sulfate groups. As described above, CNFs had larger surface area than CMFs, and the sulfate groups still remained on the surface of the CNFs exposed by Method 2. Thus, it is easier for sulfate groups to attack the hydroxyl groups of CNFs than 
those of CMFs. As a result, in Method 2, CMFs had a higher DS value than CNFs.

\section{Crystallinity}

Figure 5 presents the XRD patterns of the neat and acetylated CNFs and CMFs. In the neat CNF and $\mathrm{CMF}$ diffractograms, the diffraction peaks present at $2 \theta=15^{\circ}, 16.5^{\circ}, 22^{\circ}, 22.8^{\circ}$, and $34.5^{\circ}$ in the diffractograms were assigned to crystal planes ( $\left.\begin{array}{lll}1 & 0 & 1\end{array}\right),\left(\begin{array}{lll}1 & 0 & -1\end{array}\right),\left(\begin{array}{lll}0 & 2 & 1\end{array}\right),\left(\begin{array}{lll}0 & 0 & 2\end{array}\right)$, and (l $\left.\begin{array}{lll}0 & 4 & 0\end{array}\right)$ of the cellulose I structure, respectively. ${ }^{18}$ When the neat $\mathrm{CNF}$ diffractogram was compared with the A-CNFs diffractogram, new peaks located at $2 \theta=8^{\circ}, 10^{\circ}$, $13^{\circ}$, and $17^{\circ}$ appeared. The diffraction peaks observed at $8^{\circ}, 10^{\circ}$, and $13^{\circ}$ were assigned to the semicrystalline region of A-CNFs and ACMFs. ${ }^{11,13,24}$ Moreover, the broad peak around $17^{\circ}$ was attributed to the amorphous region of A-CNFs and A-CMFs. ${ }^{11}$ The appearance of these peaks indicated that A-CNFs and A-CMFs exhibit a more disordered structure, compared with the neat CNFs and CMFs. ${ }^{5,19}$ In Method 1, CNFs had clearer diffraction peaks located at $2 \theta=8^{\circ}, 10^{\circ}, 13^{\circ}$, and $17^{\circ}$ compared with CMFs. According to Sassi and Chanzy, it appears that acetylation starts within the amorphous region; in the next step, acetylation occurs in the crystalline region as an erosion mechanism that starts outside of the crystallite and proceeds to the center. ${ }^{25}$ As previously mentioned, CNFs exhibit a larger surface area and hydroxyl group accessibility. Because of these differences, CNFs were acetylated more easily than CMFs. The more acetylations, the more semi-crystalline regions were generated. This result is also associated with the DS values reached by Method 1 .
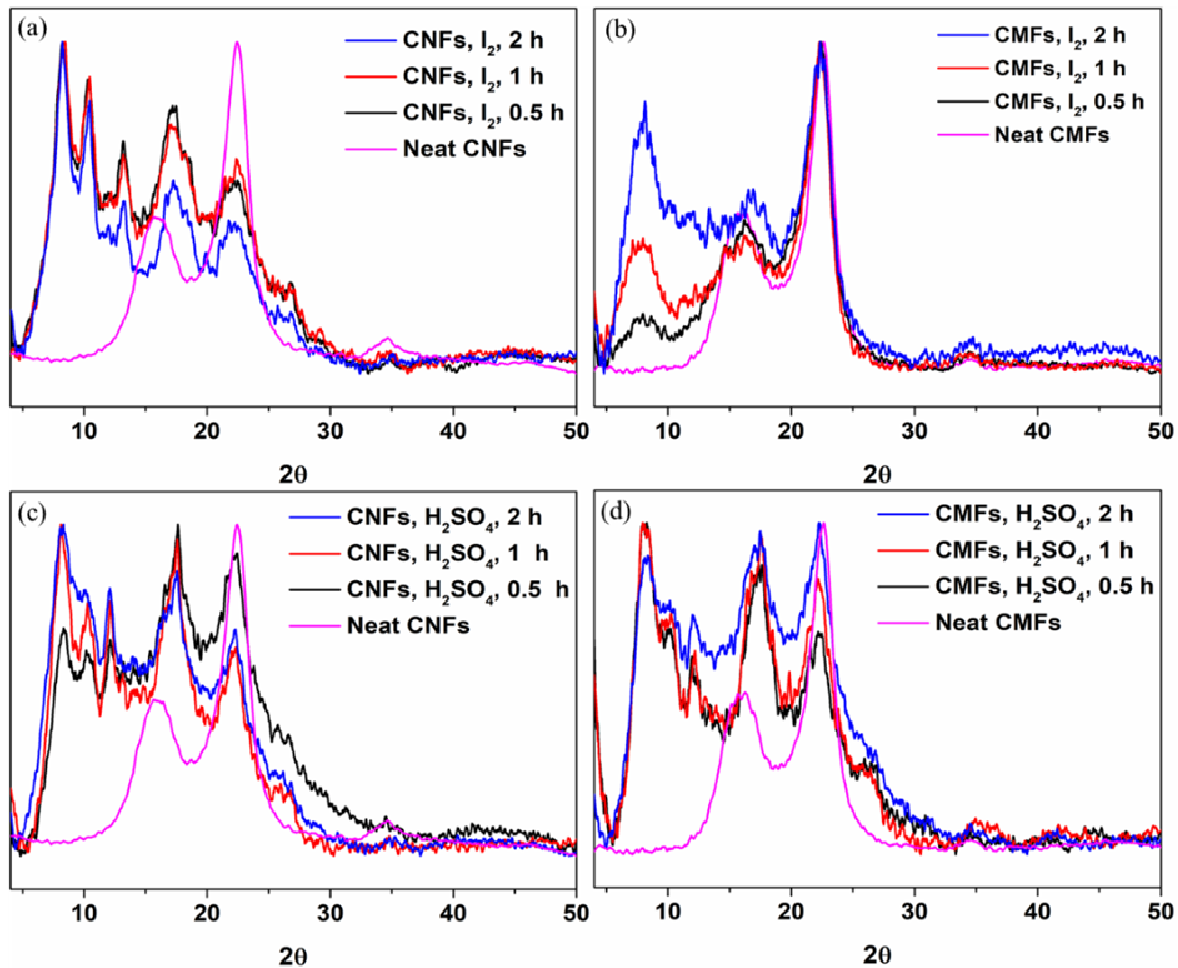

Figure 5: The XRD patterns of the a) A-CNFs treated with Method 1, b) A-CMFs treated with Method 1, c) A-CNFs treated with Method 2, d) A-CMFs treated with Method 2

In Method 2, both the CNFs and CMFs exhibit similar XRD patterns to the CNFs treated with Method 1. Unlike the CMFs treated with Method 1, the CMFs treated with Method 2 exhibit a similar XRD pattern. It is possible that this phenomenon is associated with acid hydrolysis. Since in Method 2, sulfuric acid was utilized as a catalyst, acid hydrolysis occurred, which resulted in larger surface area and higher accessibility of hydroxyl groups. As a result, CMFs are more easily acetylated than in Method 1. In Figure 6, the crystallinities of all the samples are presented. The $\mathrm{A}-\mathrm{CNF}$ a and A-CMFs exhibit lower crystallinity than the neat CNFs and CMFs. Doyle and Pethrick 
reported about a lattice spacing at $11.7 \AA$ during acetylation. ${ }^{26} \mathrm{~A}$ larger interplanar distance of $\mathrm{A}$ CNFs and A-CMFs, compared with the neat CNFs and CMFs, causes a breakdown of inter- and intramolecular hydrogen bonds. ${ }^{11,27}$ In Method 1, the crystallinities increased as the reaction time increased. In addition, the crystallinity of cellulose depends on, inter alia, its origin, processing, DP, and intermolecular and intramolecular hydrogen bond within and between the chains of the biopolymer. ${ }^{28}$ The substitution of hydroxyl groups to acetyl groups changed the crystallinity of the cellulose materials. A high DS indicates that acetylated samples have more acetyl groups, which leads to better chain packing. ${ }^{19}$ Thus, a longer reaction time results in higher crystallinity.

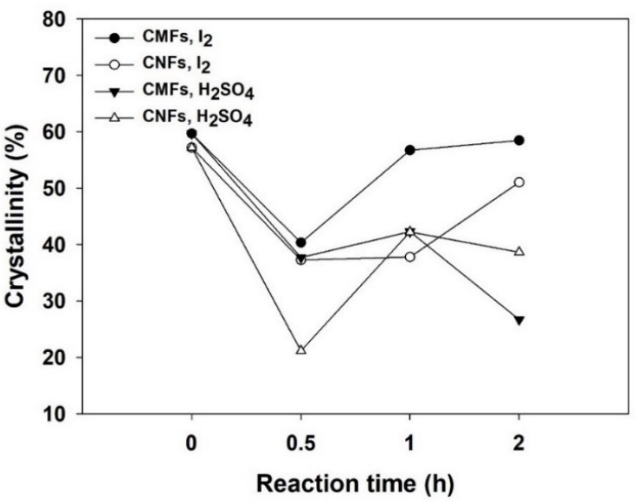

Figure 6: Crystallinity of CNFs and CMFs treated with different solvent-free acetylation methods

Furthermore, in Method 1, CMFs always exhibited higher crystallinity than CNFs, which was associated with the surface area. Since CNFs exhibited larger surface area than CMFs, more hydroxyl groups were substituted with acetyl groups. As a result, CNFs became more disordered than CMFs, and the crystallinity of CNFs was lower than that of CMFs. In Method 2, the crystallinity increased as the reaction time increased to $1 \mathrm{~h}$; however, it decreased when the reaction time increased to $2 \mathrm{~h}$. As previously mentioned, sulfuric acid was used as a catalyst, and the amount of sulfate groups increased as the reaction time increased. Thus, it is possible that the sample dissolved during the hydrolysis or expansion due to time-consuming centrifugation for washing. ${ }^{29}$ In addition, the crystallinity of CNFs in Method 2 was lower than that of CMFs at short acetylation time, as in Method 1. However, when the reaction time increased, the crystallinity of CNFs became higher than that of CMFs. As described above, CNFs exhibit a larger surface area than CMFs; thus, CNFs may have more semicrystalline regions than CMFs. As a result, CNFs exhibited lower crystallinity at short reaction time. According to Kargarzadeh et al., ${ }^{30}$ the crystallinity of nanocellulose treated with sulfuric acid increased as the reaction time increased and then decreased from a certain reaction time. This is because the amorphous region was preferentially dissolved; however, when the reaction time exceeded a certain point, the crystalline region of the nanocellulose was corroded, which indicated a decrease in crystallinity. In connection with this, the crystallinity of CNFs treated for $2 \mathrm{~h}$ could slightly decrease due to the collapse of the crystalline region, and the crystallinity of CMFs treated for $2 \mathrm{~h}$ could more significantly decrease compared with that of CNFs, as both amorphous and crystalline regions were destroyed. When Method 1 and Method 2 were compared, Method 1 was considered to be a better acetylation. This is because the Method 1 exhibits higher crystallinity, indicating that it has a more stable structure than that treated by Method 2.

\section{Thermal stability}

The TGA and derivative thermogravimetry (DTG) were employed to characterize the thermal stability of the neat and acetylated CNFs and CMFs. All thermograms are presented in Figure 7 and Figure 8. In Table 2, the peak temperature $\left(T_{p}\right)$ at the maximum DTG is presented. The TGA curves of the neat samples of both Method 1 and Method 2 exhibited weight loss from room temperature to $120{ }^{\circ} \mathrm{C}$. Conversely, the acetylated samples did not exhibit a significant difference in terms of their thermal degradation. The weight loss around $100{ }^{\circ} \mathrm{C}$ was caused by water desorption in the neat samples; however, the acetylated samples exhibited 


\section{SEONGSU PARK et al.}

no peaks, indicating $\mathrm{A}-\mathrm{CNF}$ and $\mathrm{A}-\mathrm{CMFs}$ are more hydrophobic. ${ }^{11,12}$ The $T_{p}$ value of the acetylated samples in Method 1 was higher than that of the neat samples. This indicates that the acetylated samples were more thermally stable. The increase in the thermal stability of the acetylated samples may be due to the decrease in the moisture content that accelerates the hydrolytic cleavage and substitution of hydroxyl groups with more stable chemical groups. ${ }^{14,31}$
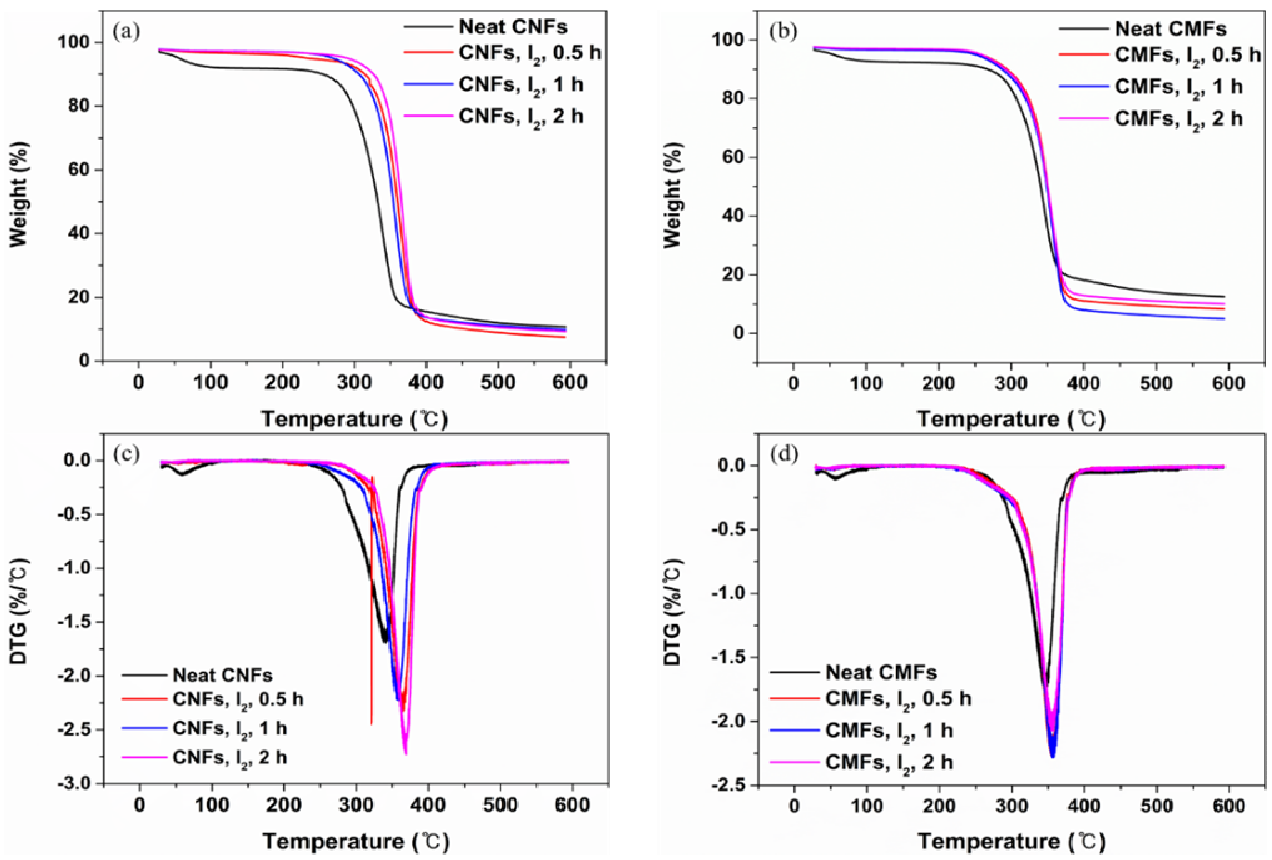

Figure 7: TGA curves of (a) CNFs and (b) CMFs treated with Method 1 and DTG curves of (c) CNFs and (d) CMFs treated with Method 1
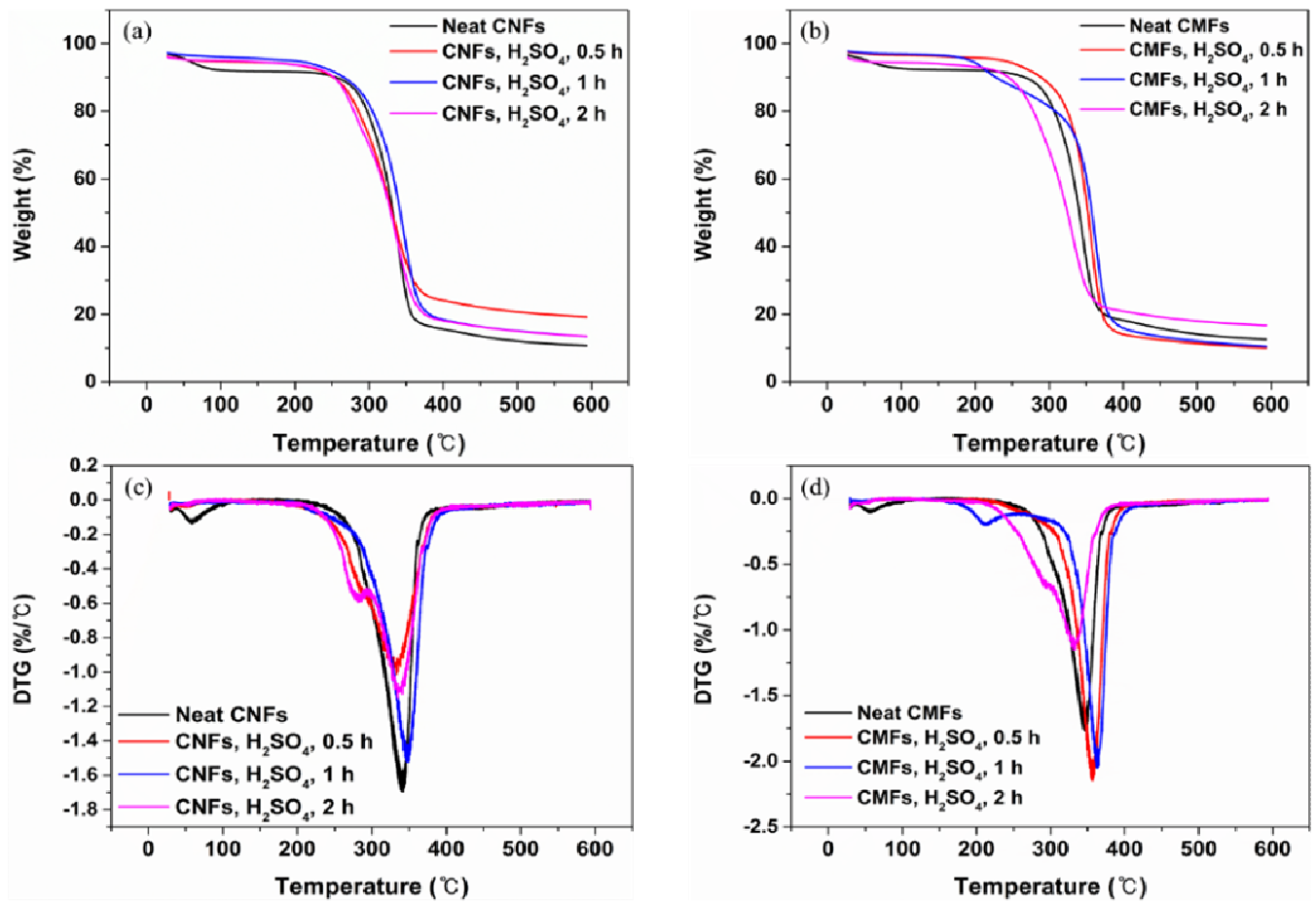

Figure 8: TGA curves of (a) CNFs and (b) CMFs treated with Method 2 and DTG curves of (c) CNFs and (d) CMFs treated with Method 2 
Table 2

$\mathrm{T}_{\mathrm{p}}$ value of CNFs and CMFs treated with different solvent-free acetylation methods

\begin{tabular}{|c|c|c|c|}
\hline Cellulose materials & Catalyst types & Time (h) & $\mathrm{T}_{\mathrm{p}}\left({ }^{\circ} \mathrm{C}\right)$ \\
\hline Neat CNFs & - & - & 340.2 \\
\hline \multirow{6}{*}{ A-CNFs } & \multirow{3}{*}{$\mathrm{I}_{2}$} & 0.5 & 365 \\
\hline & & 1 & 358.8 \\
\hline & & 2 & 369.3 \\
\hline & \multirow{3}{*}{$\mathrm{H}_{2} \mathrm{SO}_{4}$} & 0.5 & 329.6 \\
\hline & & 1 & 348.8 \\
\hline & & 2 & 334.2 \\
\hline Neat CMFs & - & - & 346.2 \\
\hline \multirow{6}{*}{ A-CMFs } & \multirow{3}{*}{$\mathrm{I}_{2}$} & 0.5 & 356.5 \\
\hline & & 1 & 356.8 \\
\hline & & 2 & 355.8 \\
\hline & \multirow{3}{*}{$\mathrm{H}_{2} \mathrm{SO}_{4}$} & 0.5 & 356.9 \\
\hline & & 1 & 363 \\
\hline & & 2 & 333.6 \\
\hline
\end{tabular}

Tingaut et al. reported that the excessive number of acetyl groups resulted in decreased crystallinity and poor thermal stability. ${ }^{15}$ The CNFs treated by Method 1 exhibited a decrease in the $T_{p}$ value when the reaction time was $1 \mathrm{~h}$ and then it increased again when the reaction time was $2 \mathrm{~h}$. The crystallinity of CNFs treated by Method 1 did not decrease due to the increase in the acetyl group. However, as reported by Tingaut et al., ${ }^{15}$ the thermal stability was lower when the reaction time was $1 \mathrm{~h}$ than when the reaction time was $0.5 \mathrm{~h}$. This phenomenon could be associated with an increase in crystallinity. Since the temperature range $220^{\circ} \mathrm{C}$ $390{ }^{\circ} \mathrm{C}$ is the point at which the crystalline region is destroyed and the degree of polymerization starts to decrease simultaneously, thermal stability would have increased in the case of the $2 \mathrm{~h}$ reaction time, suggesting higher crystallinity. ${ }^{32}$ When CNFs and CMFs treated with Method 1 were compared, the thermal stability of CNFs was found to be higher than that of CMFs. This finding may be due to two reasons: the first reason is that CNFs exhibit a more thermodynamically stable cellulose $\mathrm{I}_{\beta}$ structure than CMFs. ${ }^{23}$ The second reason is that CNFs have a larger surface area and undergo more acetylation, which could also be related to the DS.

Unlike Method 1, Method 2 demonstrated that broad peaks occur around $200{ }^{\circ} \mathrm{C}$ and between $250{ }^{\circ} \mathrm{C}$ and $300{ }^{\circ} \mathrm{C}$. These two broad peaks are associated with the sulfate groups, which were reported by Kim et al. ${ }^{33}$ and Roman and Winter. ${ }^{29}$ The generation of these peaks is attributed to the presence of sulfate groups, as it affects not only the amorphous region of the samples, but also the ends of the internal chains of the samples. ${ }^{29}$ Moreover, the $T_{p}$ value of the acetylated samples is sometimes lower than that of the neat samples. This phenomenon is due to the sulfate groups. Since the sulfate groups still remain during the acetylation process, even after the washing step, ${ }^{21}$ the remaining sulfate groups act as a catalyst, which results in poor stability. ${ }^{13}$ Furthermore, Method 2 exhibited the highest thermal stability when the reaction time was $1 \mathrm{~h}$. This can be explained by two reasons. First, as previously mentioned, since $220{ }^{\circ} \mathrm{C}-390{ }^{\circ} \mathrm{C}$ is the point where the crystalline region is destroyed, ${ }^{32}$ the 1 h-treated sample exhibiting higher crystallinity would have higher thermal stability. Second, when the reaction time is $2 \mathrm{~h}$, more sulfate groups would remain in the sample and would reduce the thermal stability. ${ }^{21} \mathrm{In}$ addition, it could be seen that CMFs had a higher $\mathrm{T}_{\mathrm{p}}$ value when compared with the CNFs and CMFs treated with Method 2. This phenomenon is related to the surface area of CMFs and CNFs. Since CNFs have a larger surface area, they are more susceptible to sulfate groups, and thus, more $\mathrm{COO}^{-}$ will form, hence resulting in the reduction of thermal stability. This can also be related to the low DS of CNFs. When Method 1 and Method 2 were compared, Method 1 was considered to be a better acetylation. This is because Method 1 leads to higher $T_{p}$ value, suggesting greater thermal stability than in the case of Method 2.

\section{Contact angle}

To compare the wettability of the samples, the CAs were measured. Figure 9 presents a graph showing the CA of the sample treated with Method 1 and Method 2. Except for the CMFs treated with Method 2, all CAs increased with an increase in the reaction time. As the reaction time increases, the 
DS also increases and more acetyl groups are formed. Thus, cellulose hydrophilicity was transformed into hydrophobicity. ${ }^{8}$ The decrease in the CA of CMFs treated with Method 2 was similar to that reported by Rodionova et al. ${ }^{16}$ The CA decreased as the reaction time increased. This result is probably associated with the sulfate groups, and it is thought that the longer the reaction time, the more sulfate groups are formed. When Method 1 and Method 2 were compared, Method 1 was considered to be a better acetylation. This is because Method 1 leads to higher $\mathrm{CA}$ and no decrease during the reaction time, indicating that the sample has higher wettability than in the case of Method 2.
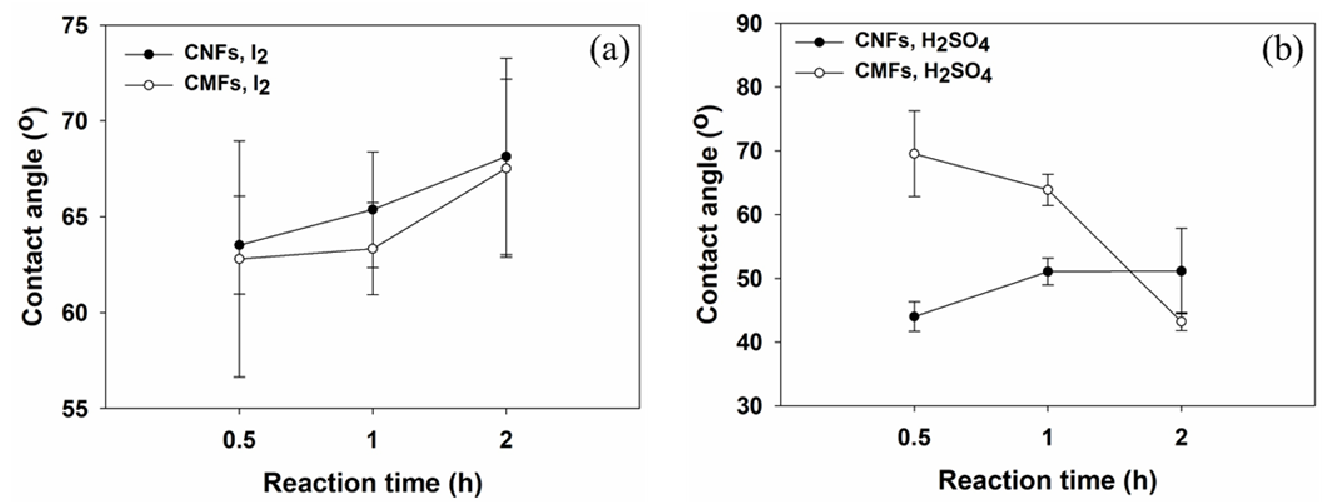

Figure 9: CAs of (a) CNFs and CMFs treated with Method 1, (b) CNFs and CMFs treated with Method 2

\section{CONCLUSION}

Two solvent-free acetylation methods were employed to convert the hydrophilicity of CNFs and CMFs to hydrophobicity. The following conclusions were drawn from this study.

1. The peak of the ATR-IR spectra confirmed that the samples had been acetylated, and the DS value increased as the reaction time increased. Moreover, the DS of Method 1 was higher for CNFs, whereas the DS of Method 2 was higher in CMFs; when the DS of Method 1 and that of Method 2 were compared, the DS in Method 2 was found to be higher.

2. The XRD analysis revealed that the acetylation converted the crystalline to the semicrystalline parts and then led to the formation of the amorphous structure. Moreover, the crystallinity of the acetylated samples was low, compared with that of the neat samples.

3. In Method 1, the crystallinity increased as the reaction time increased; however, in Method 2, the maximum value was obtained when the reaction time was $1 \mathrm{~h}$ and decreased when the reaction time was $2 \mathrm{~h}$. Furthermore, in the case of Method 1 higher crystallinity was reached than by Method 2 .

4. In Method 1, the thermal stability was increased, but in Method 2, the thermal stability was inferior in some cases. The CNFs in Method 1 exhibited higher thermal stability than CMFs, whereas the CMFs in Method 2 exhibited higher thermal stability than CNFs.
5. The CA increased as the reaction time increased, except for the CMFs treated with Method 2, indicating that the samples were modified to hydrophobic.

6. When Method 1 and Method 2 were compared, Method 1 led to better properties than Method 2. In other words, Method 1 induced greater crystallinity, thermal stability, and wettability than Method 2.

ACKNOWLEDGEMENTS: This work is supported by the Korea Forestry Promotion Institute (Grant No. 2020225B10-2022C01).

\section{REFERENCES}

P. Phanthong, P. Reubroycharoen, X. Hao, G. Xu, A. Abudula et al., Carbon Resour. Convers., 1, 32 (2018), https://doi.org/10.1016/j.crcon.2018.05.004

2 O. Nechyporchuk, M. N. Belgacem and J. Bras, Ind. Crop. Prod., 93, 2 (2016), http://dx.doi.org/10.1016/j.indcrop.2016.02.016

3 A. Sharma, M. Thakur, M. Bhattacharya, T. Mandal and S. Goswami, Biotechnol. Reports, 21, e00316 (2019), https://doi.org/10.1016/j.btre.2019.e00316

4 E. Quintana, M. Ago, C. Valls, M. B. Roncero and O. J. Rojas, Cellulose, 25, 5323 (2018), https://doi.org/10.1007/s10570-018-1947-4

5 C. M. Popescu, P. T. Larsson, N. Olaru and C. Vasile, Carbohyd. Polym., 88, $530 \quad$ (2012), https://doi.org/10.1016/j.carbpol.2011.12.046

6 A. Lepetit, R. Drolet, B. Tolnai, R. Zerrouki and D. Montplaisir, J. Appl. Polym. Sci., 134, 1 (2017), 
https://doi.org/10.1002/app.44933

7 P. B. Eranna and K. K. Pandey, Holzforschung, 66, 967 (2012), https://doi.org/10.1515/hf-2011-0223

8 M. El Boustani, F. Brouillette, G. Lebrun and A. Belfkira, J. Appl. Polym. Sci., 132, 1 (2015), https://doi.org/10.1002/app.42247

9 R. C. Sun, X. F. Sun, J. X. Sun and Q. K. Zhu, Comptes Rendus Chim., 7, $125 \quad$ (2004), https://doi.org/10.1016/j.crci.2003.10.010

10 W. Li, G. Cai and P. Zhang, J. Mater. Sci., 54, 8047 (2019), https://doi.org/10.1007/s10853-019-03471-2

11 J. Chen, J. Xu, K. Wang, X. Cao and R. Sun, Carbohyd. Polym., 137, $685 \quad$ (2016), https://doi.org/10.1016/j.carbpol.2015.11.034

12 A. Ashori, M. Babaee, M. Jonoobi and Y. Hamzeh, Carbohyd. Polym., 102, $369 \quad$ (2014), https://doi.org/10.1016/j.carbpol.2013.11.067

13 R. F. S. Barbosa, A. G. Souza, F. F. Ferreira and D. S. Rosa, Carbohyd. Polym., 218, 208 (2019), https://doi.org/10.1016/j.carbpol.2019.04.072

14 M. V. G. Zimmermann, M. P. da Silva, A. J. Zattera and R. M. Campomanes Santana, J. Appl. Polym. Sci., 134, 1 (2017), https://doi.org/10.1002/app.44760

15 P. Tingaut, T. Zimmermann and F. Lopez-Suevos, Biomacromolecules, $11, \quad 454 \quad$ (2010), https://doi.org/10.1021/bm901186u

16 G. Rodionova, M. Lenes, Ø. Eriksen and $\varnothing$. Gregersen, Cellulose, 18, $127 \quad$ (2011), https://doi.org/10.1007/s10570-010-9474-y

17 E. S. Wibowo and B. D. Park, Macromol. Res., 28, 615 (2020), https://doi.org/10.1007/s13233-020-8076-2

18 S. Park, J. O. Baker, M. E. Himmel, P. A. Parilla and D. K. Johnson, Biotechnol. Biofuels, 3, 1 (2010), https://doi.org/10.1186/1754-6834-3-10

19 H. S. Barud, A. M. de Araújo Júnior, D. B. Santos, R. M. N. de Assunção, C. S. Meireles et al., Thermochim. Acta, 471, 61 (2008), https://doi.org/10.1016/j.tca.2008.02.009

${ }^{20}$ T. Kakko, A. W. T. King and I. Kilpeläinen, Cellulose, 24, 5341 (2017), https://doi.org/10.1007/s10570-017-1521-5

21 N. Wang, E. Ding and R. Cheng, Polymer (Guildf), 48 , 3486

(2007),
22 T. I. M. Ragab, A. A. F. Wasfy, H. Amer, A. ElGendi, M. S. Abdel-Hady et al., J. Appl. Sci., 14, 3424 (2014), https://scialert.net/abstract/?doi=jas.2014.3424.3435

23 R. Kose, I. Mitani, W. Kasai and T. Kondo, Biomacromolecules, $\quad \mathbf{1 2}, 716 \quad$ (2011), https://doi.org/10.1021/bm1013469

24 X. Sun, C. Lu, W. Zhang, D. Tian and X. Zhang, Carbohyd. Polym., 98, $405 \quad$ (2013), http://dx.doi.org/10.1016/j.carbpol.2013.05.089

25 J. F. Sassi and H. Chanzy, Cellulose, 2, 111 (1995), https://doi.org/10.1007/BF00816384

26 S. E. Doyle and R. A. Pethrick, J. Appl. Polym. Sci., 33 , 95

(1987),

https://doi.org/10.1002/app.1987.070330109

27 G. R. Filho, S. F. Da Cruz, D. Pasquini, D. A. Cerqueira, V. D. S. Prado et al., J. Memb. Sci., 177, 225 (2000), https://doi.org/10.1016/S0376-7388(00)00469-5

28 T. Heinze and O. A. El Seoud, "Cellulose Derivatives. Sythesis, Structure and Properties", Springer Series on Polymer and Composite Materials Cellulose Derivatives, Springer International Publishing AG, 2018, https://doi.org/10.1007/9.78-3-319-73168-1

29 M. Roman and W. T. Winter, Biomacromolecules, 5, 1671 (2004), https://doi.org/10.1021/bm034519+

30 H. Kargarzadeh, I. Ahmad, I. Abdullah, A. Dufresne, S. Y. Zainudin et al., Cellulose, 19, 855 (2012), https://doi.org/10.1007/s10570-012-9684-6

31 N. Lin, J. Huang, P. R. Chang, J. Feng and J. Yu, Carbohyd. Polym., 83, $1834 \quad$ (2011), http://dx.doi.org/10.1016/j.carbpol.2010.10.047

32 W. Hu, S. Chen, Q. Xu and H. Wang, Carbohyd. Polym., $\quad \mathbf{8 3}, \quad 1575 \quad$ (2011) http://dx.doi.org/10.1016/j.carbpol.2010.10.016

33 D. Y. Kim, Y. Nishiyama, M. Wada and S. Kuga, Cellulose, $\quad \mathbf{8 ,} \quad 29 \quad$ (2001), https://doi.org/10.1023/A:1016621103245

https://doi.org/10.1016/j.polymer.2007.03.062 